\title{
Supplement to: Normalizing constants of log-concave densities
}

\author{
Nicolas Brosse \\ Centre de Mathématiques Appliquées, UMR 7641, Ecole Polytechnique, France. \\ e-mail: nicolas.brosse@polytechnique.edu \\ Alain Durmus \\ Ecole Normale Supérieure CMLA, 61 Av. du Président Wilson 94235 Cachan Cedex, France. \\ e-mail: alain.durmus@cmla.ens-cachan.fr \\ and

\section{Éric Moulines} \\ Centre de Mathématiques Appliquées, UMR 7641, Ecole Polytechnique, France. \\ e-mail: eric.moulines@polytechnique.edu
}

\section{S1. Proofs of Section 2.1}

\section{S1.1. Proof of Lemma 10}

In this Section, the proof for the case $i=M-1$ of Lemma 10 is dealt with. Note that $a_{M-1}=$ $\left(2 \sigma_{M-1}^{2}\right)^{-1}$. By Propositions 2 and 4 , to check condition-ii) of Lemma 9, it is then sufficient for $\gamma_{M-1}, n_{M-1}, N_{M-1}$ to satisfy,

$$
\begin{aligned}
& \frac{4 d}{n_{M-1} m_{M-1} \kappa_{M-1} \gamma_{M-1}} \exp \left(-N_{M-1} \frac{\kappa_{M-1} \gamma_{M-1}}{2}\right) \\
& +2 \kappa_{M-1}^{-1}\left(A_{M-1,0} \gamma_{M-1}+A_{M-1,1} \gamma_{M-1}^{2}\right) \leq \frac{\eta^{2} \sigma_{M-1}^{4}}{C_{M-1,2}+C_{M-1,0} C_{M-1,1}}, \\
& \frac{8 C_{M-1,0} C_{M-1,1}}{\kappa_{M-1}^{2} n_{M-1} \gamma_{M-1}}\left(1+\frac{2}{\kappa_{M-1} n_{M-1} \gamma_{M-1}}\right) \leq \sigma_{M-1}^{4} \eta^{2} .
\end{aligned}
$$

Then (S1) and (S2) are satisfied if,

$$
\begin{aligned}
& 2 L_{M-1}^{2} \kappa_{M-1}^{-1} d \gamma_{M-1}+4 d L_{M-1}^{4} \kappa_{M-1}^{-1} m_{M-1}^{-1} \gamma_{M-1}^{2} \leq \frac{\eta^{2}}{4} \frac{\kappa_{M-1} m_{M-1} \sigma_{M-1}^{4}}{\alpha_{M-1} d} \\
& \frac{1}{n_{M-1}}\left(1+\frac{2}{\kappa_{M-1} n_{M-1} \gamma_{M-1}}\right) \leq \frac{\eta^{2} \sigma_{M-1}^{4} \kappa_{M-1}^{3} \gamma_{M-1}}{8 \beta_{M-1} d} \\
& -2\left(\kappa_{M-1} \gamma_{M-1}\right)^{-1} \log \left(\frac{\eta^{2} \sigma_{M-1}^{4} m_{M-1}^{2} n_{M-1} \kappa_{M-1} \gamma_{M-1}}{8 \alpha_{M-1} d^{2}}\right)=\bar{N}_{M-1} \leq N_{M-1} .
\end{aligned}
$$


Denote by $\bar{\gamma}_{M-1}$ and $\bar{n}_{M-1}^{-1}$ the positive roots associated to (S3) and (S4) seen as equalities. We have:

$$
\begin{aligned}
& \bar{\gamma}_{M-1}=4^{-1} L_{M-1}^{-2} m_{M-1}\left(-1+\sqrt{1+\frac{\eta^{2} \kappa_{M-1}^{2} \sigma_{M-1}^{4}}{\alpha_{M-1} d^{2}}}\right), \\
& \bar{n}_{M-1}^{-1}=4^{-1} \kappa_{M-1} \gamma_{M-1}\left(-1+\sqrt{1+\frac{\eta^{2} \kappa_{M-1}^{2} \sigma_{M-1}^{4}}{\beta_{M-1} d}}\right) .
\end{aligned}
$$

Note that for (S3) and (S4) to hold, it suffices that $\gamma_{M-1} \leq \bar{\gamma}_{M-1}$ and $n_{M-1} \geq \bar{n}_{M-1}$. We now lower bound $\bar{\gamma}_{M-1}$ and upper bound $\bar{n}_{M-1}$.

Using that $t \geq 0, \sqrt{1+t} \geq 1+2^{-1} t(1+t)^{-1 / 2}$ for $t=\left(\eta^{2} \kappa_{M-1}^{2} \sigma_{M-1}^{4}\right) /\left(\alpha_{M-1} d^{2}\right)$ and $\kappa_{M-1} \sigma_{M-1}^{2} d^{-1} \geq 2, \alpha_{M-1} \geq 4$ concludes that if (43) holds then $\gamma_{M-1} \leq \bar{\gamma}_{M-1}$. The fact that $\gamma_{M-1} \leq\left(m_{M-1}+L_{M-1}\right)^{-1}$ can be checked by simple algebra.

Then using that $\sqrt{1+t} \geq 1+2^{-1} t(1+t)^{-1 / 2}$ for $t=\left(\eta^{2} \kappa_{M-1}^{2} \sigma_{M-1}^{4}\right) /\left(\beta_{M-1} d\right)$ and $\kappa_{M-1} \sigma_{M-1}^{2} \geq$ $10, \beta_{M-1} \geq 1$ concludes that if (44) holds then $n_{M-1} \geq \bar{n}_{M-1}$. Finally, by (44), we get

$$
\bar{N}_{M-1} \leq\left(\kappa_{M-1} \gamma_{M-1}\right)^{-1} \log (7 / 3),
$$

which concludes that (45) implies (S5).

\section{S1.2. Proof of Lemma 11}

Let $k \in\{0, \ldots, K-1\}$ and $i \in \mathcal{I}_{k}$. Assume that $\gamma_{i} \leq\left(m_{i}+L_{i}\right)^{-1}$. The proof of Lemma 10 only needs to be slightly adapted. More precisely, Proposition 3 is applied instead of Proposition 2. By (26) and (27), we have

$$
B_{i, 0} \leq 3^{-1} d \kappa_{i}^{-1}\left(d \tilde{L}^{2}+10 L_{i}^{4} m_{i}^{-1}\right), \quad B_{i, 1} \leq(25 / 12) d L_{i}^{4} m_{i}^{-1} .
$$

It is sufficient for $\gamma_{i}, n_{i}, N_{i}$ to satisfy (107) and (108) with $A_{i, 0} \gamma_{i}+A_{i, 1} \gamma_{i}^{2}$ replaced by $B_{i, 0} \gamma_{i}^{2}+$ $B_{i, 1} \gamma_{i}^{3}$. The counterpart of (109) is then

$$
\frac{1}{3 \kappa_{i}}\left(d \tilde{L}^{2}+10 L_{i}^{4} m_{i}^{-1}\right) \gamma_{i}^{2}+\frac{25}{12} L_{i}^{4} m_{i}^{-1} \gamma_{i}^{3} \leq \frac{\eta^{2} \kappa_{i} m_{i} \sigma_{i}^{4}}{16 K^{2} \alpha_{i} d^{2}} .
$$

Since $\gamma_{i} \leq 1 /\left(m_{i}+L_{i}\right)$ and $\kappa_{i} \leq L_{i}$, we have

$$
\left(3 \kappa_{i}\right)^{-1}\left(d \tilde{L}^{2}+10 L_{i}^{4} m_{i}^{-1}\right) \geq(25 / 12) L_{i}^{4} m_{i}^{-1} \gamma_{i},
$$

which establishes that if (46) holds, then (S9) is satisfied. $\gamma_{i} \leq\left(m_{i}+L_{i}\right)^{-1}$ can be checked by simple algebra. For $i=M-1$, the conclusion follows from $m_{M-1} \sigma_{M-1}^{2} d^{-1} \geq 2$ because $\sigma_{M-1}^{2} \geq(2 d+7) / m$.

\section{S2. Proofs of Section 2.2}

First, we state a technical lemma that gathers useful bounds. We recall that $M$ is defined in this Section by (56),

$$
M=\inf \left\{i \geq 1: \sigma_{i-1}^{2} \geq D^{2}\right\} .
$$


Lemma S1. Assume $\boldsymbol{H} 1$ and $\boldsymbol{H} 2(m)$ for $m \geq 0$. Let $\left\{\sigma_{i}^{2}\right\}_{i=0}^{M-1}$ defined by (54) for $\sigma_{0}^{2}$ given in (31) and $M$ in (56).

1. $K \leq\left\lceil(1 / \log (2)) \log \left(\sigma_{0}^{-2} \rho^{-2} d^{2}(\tau+1)^{2}\right)\right\rceil$ where $K$ is defined in (39).

2. For $k \in\{0, \ldots, K-1\}$ and $i \in \mathcal{I}_{k}, 2^{k+1} \sigma_{0}^{2} a_{i}\left|\mathcal{I}_{k}\right| \leq 1$ where $a_{i}$ is defined in (37) (with $m=0)$ and $\mathcal{I}_{k}$ in $(38)$. As a consequence, $\left|\mathcal{I}_{k}\right| \leq 4(d+4)$.

3. For $i \in\{0, \ldots, M-1\}, \kappa_{i} \sigma_{i}^{2} \in[1,2]$.

4. $\sigma_{M-1}^{2} \in\left[D^{2},(10 / 9) D^{2}\right]$.

5. For all $i \in\{0, \ldots, M-1\}$ and $\gamma_{i} \leq 1 /\left(m_{i}+L_{i}\right)$, there exist $\alpha_{i} \in[4,14]$ and $\beta_{i} \in[1,10]$ such that $C_{i, 2}+C_{i, 0} C_{i, 1}=\alpha_{i} d m_{i}^{-1}$ and $C_{i, 0} C_{i, 1}=\beta_{i} d \kappa_{i}^{-1}$ where $C_{i, 0}, C_{i, 1}, C_{i, 2}$ and $\kappa_{i}$ are given in (23) and (14) respectively.

6. For all $i \in\{0, \ldots, M-1\}, 0<A_{i, 1} \leq 4 d L_{i}^{4} \kappa_{i}^{-1} m_{i}^{-1}$, where $L_{i}, m_{i}$ and $\kappa_{i}$ are given in (13) and (14) respectively.

Proof. The proofs of 1,2,5,6 are identical to the ones of Lemma 23.

3. $\kappa_{i} \sigma_{i}^{2}=\left(2 L_{i}\right) /\left(m_{i}+L_{i}\right)$.

4. By definition of $M, \sigma_{M-2}^{2} \leq D^{2}$ and $a_{M-2} \leq \sigma_{M-2}^{-2} /\{4(d+4)\}$. By (6), we get:

$$
\sigma_{M-1}^{-2}=\sigma_{M-2}^{-2}-2 a_{M-2} \geq \sigma_{M-2}^{-2}\left(1-\frac{1}{2(d+4)}\right)
$$

that is $\sigma_{M-1}^{2} \leq(10 / 9) \sigma_{M-2}^{2} \leq(10 / 9) D^{2}$.

\section{S2.1. Proof of Lemma 15}

Let $k \in\{0, \ldots, K-1\}$ and $i \in \mathcal{I}_{k}$. Assume that $\gamma_{i} \leq\left(m_{i}+L_{i}\right)^{-1}$. The proof follows the same lines as the one in Section 5.3.2. By Lemma S1-5 and Lemma S1-6, to check condition-i) of Lemma 9, it suffices that $\gamma_{i} \leq \bar{\gamma}_{i}, n_{i} \geq \bar{n}_{i}$ and $N_{i}$ satisfies (111), where $\bar{\gamma}_{i}$ is defined in (112) and $\bar{n}_{i}$ in (113).

Using that $\sqrt{1+t} \geq 1+2^{-1} t(1+t)^{-1 / 2}$ for $t=\left(\eta^{2} \kappa_{i}^{2} \sigma_{i}^{4}\right) /\left(4 \alpha_{i} K^{2} d^{2}\right)$ and by Lemma S1-3, concludes that if (57) holds then $\gamma_{i} \leq \bar{\gamma}_{i} \cdot \gamma_{i} \leq\left(m_{i}+L_{i}\right)^{-1}$ can be checked by simple algebra.

By (37) (with $m=0)$ and the definition of $\mathcal{I}_{k}, a_{i} \leq \sigma_{i}^{-2} /\{4(d+4)\}$,

$$
\bar{n}_{i}^{-1} \geq 4^{-1} \kappa_{i} \gamma_{i}\left(-1+\sqrt{1+\frac{\eta^{2} \kappa_{i}^{2} \sigma_{i}^{4}(d+4)}{K \beta_{i} d}}\right) .
$$

Using that $\sqrt{1+t} \geq 1+2^{-1} t(1+t)^{-1 / 2}$ for $t=\left(\eta^{2} \kappa_{i}^{2} \sigma_{i}^{4}\right) /\left(4 \alpha_{i} K^{2} d^{2}\right)$ and by Lemma S1-3, concludes that if (58) holds then $n_{i} \geq \bar{n}_{i}$. Finally, by (58), if (59) holds, (111) is satisfied.

The case $i=M-1$ is different because $\bar{g}_{M-1}$ is Lipschitz. Assume $\gamma_{M-1} \leq\left(m_{M-1}+L_{M-1}\right)^{-1}$. [1, section 2.1] entails that condition-ii) of Lemma 9 is satisfied if

$$
\begin{aligned}
&\left\|\bar{g}_{M-1}\right\|_{\text {Lip }}^{2}\left\{\frac{4 d}{n_{M-1} m_{M-1} \kappa_{M-1} \gamma_{M-1}} \exp \left(-N_{M-1} \frac{\kappa_{M-1} \gamma_{M-1}}{2}\right)\right.\left.+2 \kappa_{M-1}^{-1}\left(A_{M-1,0} \gamma_{M-1}+A_{M-1,1} \gamma_{M-1}^{2}\right)\right\} \leq \eta^{2}, \\
& \frac{8\left\|\bar{g}_{M-1}\right\|_{\text {Lip }}^{2}}{\kappa_{M-1}^{2} n_{M-1} \gamma_{M-1}}\left\{1+\frac{2}{n_{M-1} \kappa_{M-1} \gamma_{M-1}}\right\} \leq \eta^{2} .
\end{aligned}
$$


Using $\left\|\bar{g}_{M-1}\right\|_{\text {Lip }}^{2} \leq\left(\sigma_{M-1}^{-2} \mathrm{e}\right)$ and (24), Lemma S1-6 for $i=M-1$, it is sufficient for $\gamma_{M-1}, n_{M-1}, n_{M-1}$ to satisfy

$$
\begin{aligned}
2 L_{M-1}^{2} \kappa_{M-1}^{-1} d \gamma_{M-1}+4 d L_{M-1}^{4} \kappa_{M-1}^{-1} m_{M-1}^{-1} \gamma_{M-1}^{2} & \leq(4 \mathrm{e})^{-1} \kappa_{M-1} \eta^{2} \sigma_{M-1}^{2} \\
n_{M-1}^{-1}\left(1+2\left(\kappa_{M-1} \gamma_{M-1} n_{M-1}\right)^{-1}\right) & \leq \frac{\eta^{2} \kappa_{M-1}^{2} \sigma_{M c-1}^{2} \gamma_{M-1}}{8 \mathrm{e}} \\
-2 \frac{\log \left((8 \mathrm{e} d)^{-1} n_{M-1} \kappa_{M-1} \gamma_{M-1} \eta^{2}\right)}{\kappa_{M-1} \gamma_{M-1}} & =\bar{N}_{M-1} \leq N_{M-1}
\end{aligned}
$$

Denote by $\bar{\gamma}_{M-1}, \bar{n}_{M-1}^{-1}$ the roots of (S11), (S12) seen as equalities. We have

$$
\begin{aligned}
& \bar{\gamma}_{M-1}=4^{-1} L_{M-1}^{-2} \sigma_{M c-1}^{-2}\left\{-1+\sqrt{1+\frac{\eta^{2} \kappa_{M-1}^{2} \sigma_{M-1}^{4}}{\mathrm{e} d}}\right\}, \\
& \bar{n}_{M-1}^{-1}=4^{-1} \kappa_{M-1} \gamma_{M-1}\left\{-1+\sqrt{1+\mathrm{e}^{-1} \eta^{2} \kappa_{M-1} \sigma_{M-1}^{2}}\right\} .
\end{aligned}
$$

Using that $\sqrt{1+t} \geq 1+2^{-1} t(1+t)^{-1 / 2}$ for $t=\left(\eta^{2} \kappa_{M-1}^{2} \sigma_{M-1}^{4}\right) /(\mathrm{e} d)$ and by Lemma S1-3, concludes that if (60) holds then $\gamma_{M-1} \leq \bar{\gamma}_{M-1} \cdot \gamma_{M-1} \leq\left(m_{M-1}+L_{M-1}\right)^{-1}$ can be checked by simple algebra.

Using that $\sqrt{1+t} \geq 1+2^{-1} t(1+t)^{-1 / 2}$ for $t=\mathrm{e}^{-1} \eta^{2} \kappa_{M-1} \sigma_{M-1}^{2}$ and by Lemma S1-3, concludes that if $(61)$ holds then $n_{M-1} \geq \bar{n}_{M-1}$.

Finally by (61), if (62) holds, (S13) is satisfied.

\section{S2.2. Proof of Lemma 16}

The proof is identical to the one of Lemma 11. For $k \in\{0, \ldots, K-1\}$ and $i \in \mathcal{I}_{k}$, it is sufficient for $\gamma_{i}$ to satisfy (63) by (46) and Lemma S1-3.

Regarding the case $i=M-1$, assuming that $\gamma_{M-1} \leq\left(m_{M-1}+L_{M-1}\right)^{-1}$, it is sufficient for $\gamma_{M-1}, n_{M-1}, N_{M-1}$ to satisfy (S10) with $A_{M-1,0} \gamma_{M-1}+A_{M-1,1} \gamma_{M-1}^{2}$ replaced by $B_{M-1,0} \gamma_{M-1}^{2}+$ $B_{M-1,1} \gamma_{M-1}^{3}$. The counterpart of (S11) is then,

$$
\frac{1}{3 \kappa_{M-1}}\left(d \tilde{L}^{2}+10 L_{M-1}^{4} m_{M-1}^{-1}\right) \gamma_{M-1}^{2}+\frac{25}{12} \frac{L_{M-1}^{4}}{m_{M-1}} \gamma_{M-1}^{3} \leq \frac{\eta^{2} \kappa_{M-1} \sigma_{M-1}^{2}}{4 \mathrm{e} d} .
$$

This concludes the proof with the same argument as in Section S1.2.

\section{S2.3. Proof of Theorems 12 and 13 and corollary 14}

For $i \in\{0, \ldots, M-1\}$, set $\gamma_{i}, n_{i}, N_{i}$ such that (57), (58), (59), (60), (61) and (62) are equalities. By (18), we have

$$
\text { cost }=\left(\frac{453 K}{\eta^{2}}+2 \log \left(K d^{2}\right)\right) \frac{462 K^{2} d^{2}}{\eta^{2}} \sum_{i=0}^{M-2} \kappa_{i}^{-1} L_{i}^{2} \sigma_{i}^{2}+n_{M-1}+N_{M-1} .
$$

Note that for $i \in\{0, \ldots, M-2\}$,

$$
\kappa_{i}^{-1} L_{i}^{2} \sigma_{i}^{2}=1+(3 / 2) L \sigma_{i}^{2}+\left(L^{2} / 2\right) \sigma_{i}^{4} .
$$


By Lemma S1-2, for $k \in\{0, \ldots, K-1\},\left|\mathcal{I}_{k}\right| \leq 4(d+4)$ and for $i \in \mathcal{I}_{k}, \sigma_{i}^{2} \leq 2^{k+1} \sigma_{0}^{2}$. We then have

$$
\begin{aligned}
\sum_{i=0}^{M-2} \frac{L_{i}^{2} \sigma_{i}^{2}}{\kappa_{i}} & \leq 4(d+4) \sum_{k=0}^{K-1}\left\{1+\frac{3 L}{2} 2^{k+1} \sigma_{0}^{2}+\frac{L^{2}}{2}\left(2^{k+1} \sigma_{0}^{2}\right)^{2}\right\} \\
& \leq 4(d+4)\left\{K+3 L\left(2^{K} \sigma_{0}^{2}\right)+\frac{2 L^{2}}{3}\left(2^{K} \sigma_{0}^{2}\right)^{2}\right\} .
\end{aligned}
$$

By (56) and the definition of $K,(39), 2^{K} \sigma_{0}^{2} \leq 2 D^{2}$. The expressions of $\gamma_{M-1}, n_{M-1}, N_{M-1}$ give

$$
n_{M-1}+N_{M-1}=\left(\frac{29}{\eta^{2}}+2 \log (d)\right) \frac{26 d}{\eta^{2}} \kappa_{M-1}^{-2} L_{M-1}^{2},
$$

with $\kappa_{M-1}^{-2} L_{M-1}^{2}=\left(1+2^{-1} L \sigma_{M-1}^{2}\right)^{2}$. By Lemma S1-4, we then have

$$
n_{M-1}+N_{M-1} \leq\left(\frac{29}{\eta^{2}}+2 \log (d)\right) \frac{26 d}{\eta^{2}}\left(1+\frac{5 L}{9} D^{2}\right)^{2},
$$

and (51) is established.

Assume H3. We now prove Theorem 13 and use Lemma 11 instead of Lemma 10. For $i \in$ $\{0, \ldots, M-1\}$, set $\gamma_{i}, n_{i}, N_{i}$ such that (63), (58), (59), (64), (61) and (62) are equalities. By (18) and using that for $a, b \geq 0, \sqrt{a+b} \leq \sqrt{a}+\sqrt{b}$, we have

$$
\text { cost } \leq\left(\frac{453 K}{\eta^{2}}+2 \log \left(K d^{2}\right)\right) \sqrt{\frac{7}{3}} \frac{8 K d}{\eta} \sum_{i=0}^{M-2} \frac{\sigma_{i}}{\kappa_{i}}\left(d^{1 / 2} \tilde{L}+\sqrt{10} L_{i}^{2} \sigma_{i}\right)+n_{M-1}+N_{M-1} .
$$

For $k \in\{0, \ldots, K-1\}$ and $i \in \mathcal{I}_{k}$, note that

$$
\kappa_{i}^{-1} \sigma_{i} \leq \sigma_{i}^{3}, \quad \kappa_{i}^{-1} \sigma_{i}^{2} L_{i}^{2}=1+\frac{3 L}{2} \sigma_{i}^{2}+\frac{L^{2}}{2} \sigma_{i}^{4} .
$$

Using for $k \in\{0, \ldots, K-1\},\left|\mathcal{I}_{k}\right| \leq 4(d+4)$ by Lemma S1-2 and for $i \in \mathcal{I}_{k}, \sigma_{i}^{2} \leq 2^{k+1} \sigma_{0}^{2}$, we get

$$
\begin{aligned}
\sum_{i=0}^{M-2} \frac{\sigma_{i}}{\kappa_{i}}\left(d^{1 / 2} \tilde{L}+\sqrt{10} L_{i}^{2} \sigma_{i}\right) & \leq 4(d+4) \sum_{k=0}^{K-1}\left\{d^{1 / 2} \tilde{L}\left(2^{k+1} \sigma_{0}^{2}\right)^{3 / 2}\right. \\
& \left.+\sqrt{10}\left(1+\frac{3 L}{2}\left(2^{k+1} \sigma_{0}^{2}\right)+\frac{L^{2}}{2}\left(2^{k+1} \sigma_{0}^{2}\right)^{2}\right)\right\} \\
& \leq 4(d+4)\left\{5 d^{1 / 2} \tilde{L} D^{3}+\sqrt{10}\left(K+6 L D^{2}+\frac{8 L^{2}}{3} D^{4}\right)\right\},
\end{aligned}
$$

with $2^{K} \sigma_{0}^{2} \leq 2 D^{2}$. The expressions of $\gamma_{M-1}, n_{M-1}, N_{M-1}$ give

$$
n_{M-1}+N_{M-1} \leq\left(2 \log (d)+\frac{29}{\eta^{2}}\right) \sqrt{\frac{8 \mathrm{e}}{3}} \frac{\sqrt{d}}{\eta} \frac{d^{1 / 2} \tilde{L}+\sqrt{10} L_{M-1}^{2} \sigma_{M-1}}{\kappa_{M-1}^{2} \sigma_{M-1}} .
$$

By Lemma S1-4, $\sigma_{M-1}^{2} \in\left[D^{2},(10 / 9) D^{2}\right]$. We get then

$$
\kappa_{M-1}^{-2} \sigma_{M-1}^{-1}=\frac{\left(1+(L / 2) \sigma_{M-1}^{2}\right)^{2}}{L^{2} \sigma_{M-1}} \leq \frac{1}{D L^{2}}\left(1+\frac{5 L}{9} D^{2}\right)^{2}
$$


and,

$$
\kappa_{M-1}^{-2} L_{M-1}^{2}=\left(1+\frac{L}{2} \sigma_{M-1}^{2}\right)^{2} \leq\left(1+\frac{5 L}{9} D^{2}\right)^{2}
$$

which gives,

$$
n_{M-1}+N_{M-1} \leq\left(2 \log (d)+\frac{29}{\eta^{2}}\right) \sqrt{\frac{8 \mathrm{e}}{3}} \frac{\sqrt{d}}{\eta}\left(1+\frac{5 L}{9} D^{2}\right)^{2}\left(\frac{d^{1 / 2} \tilde{L}}{D L^{2}}+\sqrt{10}\right) .
$$

(53) is established. The proof of Corollary 14 is the same as the one of Corollary 7.

\section{References}

[1] A. Durmus and E. Moulines. High-dimensional Bayesian inference via the unadjusted Langevin algorithm. May 2016. 